\title{
Caracterização das Estratégias de Qualidade de Uso que Potencializam a Utilização Contínua de Aplicativos Colaborativos de Navegação: Um estudo de caso no Waze
}

\author{
Lorena Tomagnini ${ }^{1}$, Glívia A. R. Barbosa ${ }^{1}$, Geanderson E. dos Santos ${ }^{2}$, Ismael S. \\ Silva $^{1}$
}

${ }^{1}$ Departamento de Computação - Centro Federal de Educação Tecnológica de Minas Gerais (DECOM/CEFET-MG). Av. Amazonas, 7675 - Nova Gameleira - Belo Horizonte, MG Brasil.

${ }^{2}$ Departamento de Ciência da Computação - Universidade Federal de Minas Gerais (DCC/UFMG). Av. Antônio Carlos, 6627 - Prédio do ICEx Pampulha, Belo Horizonte, MG - Brasil.

lorenagft2013@gmail.com, gliviabarbosaldecom.cefetmg.br, geanderson@dcc.ufmg.br, ismaelsantana@decom.cefetmg.br

\begin{abstract}
This study aimed to verify and characterize, through a case study in Waze, the use of quality strategies adopted by collaborative applications of navigation and the contribution these strategies in the continued use of this type of application. The results showed that the designer takes strategies considered relevant to promote a collaborative environment that maintains the user to interact continuously.
\end{abstract}

Resumo. Este trabalho buscou verificar e caracterizar, através de um estudo de caso no Waze, as estratégias de qualidades de uso adotadas por aplicativos colaborativos de navegação e a contribuição das mesmas para o uso contínuo desse tipo de aplicação. Os resultados mostraram que o projetista adota estratégias consideradas relevantes para promover um ambiente colaborativo que mantém o usuário motivado a interagir continuamente.

\section{Introdução}

A utilização de aplicativos móveis em diferentes contextos colaborativos (e.g., aplicativos sociais, educacionais e aplicativos de localização/navegação) vem crescendo e esse crescimento abre espaço para que diversos aplicativos da mesma categoria sejam disponibilizados para os usuários (e.g., aplicativos de comunicação: WhatsApp, Telegram e Hangouts) [de Oliveira et al. 2012][Google Play, 2016].

Diante dessa realidade, surge o desafio de motivar os usuários no uso constante desses aplicativos de modo que eles se mantenham fidelizados e engajados na utilização dos mesmos [de Oliveira et al. 2012]. Pensando neste aspecto, projetistas de interface e pesquisadores da Computação (e.g., [Barbosa et al. 2013][Silva et al. 2015]) têm investigado a aplicabilidade de adotar qualidades de uso (e.g., a colaboração, sociabilidade e gamificação) nos modelos de interface e interação de aplicativos colaborativos com o 
intuito de incentivar o uso contínuo desse tipo de aplicação.

Uma categoria de aplicativos colaborativos que vem ganhando força, por seu uso constante, é a de aplicativos de navegação. Nesses aplicativos, o usuário pode solicitar o fornecimento de rotas, os trajetos com menor tempo de deslocamento, a visualização de mapas em tempo real, entre outras funcionalidades [Tarouco, 2013][Google Play, 2016]. Dentro deste domínio, o aplicativo Waze se destaca com mais de 50 milhões de usuários ao redor do mundo [Waze, 2016], sendo que, no Brasil, esse número ultrapassa os 6 milhões de usuários, fazendo do país o $2^{\circ}$ no ranking de utilização do Waze [O Globo, 2013][Tarouco, 2013][Google Play, 2016].

Motivados pelo desafio de manter os usuários engajados na utilização de aplicativos móveis colaborativos, considerando a grande adoção dos aplicativos de navegação e sua importância no uso cotidiano e, pelo fato do Waze ser um fenômeno em termos de adoção mundial, o objetivo deste trabalho consiste em analisar e caracterizar como o aplicativo Waze tem motivado seu uso contínuo, de forma a: (1) identificar e demonstrar as estratégias comunicadas na interface que podem atuar como fatores motivacionais para este uso contínuo e (2) apresentar quais estratégias de fato contribuem para o engajamento dos usuários, considerando a perspectiva dos mesmos.

Os resultados indicaram que o Waze utiliza estratégias, tais como, usabilidade, comunicabilidade, acessibilidade, colaboração, sociabilidade e gamificação, que são consideradas relevantes para potencializar a experiência satisfatória dos usuários e, sob a perspectiva dos usuários participantes dessa pesquisa, essas estratégias contribuem para que eles se mantenham engajados no uso do aplicativo. Sendo assim, esse trabalho apresenta contribuições práticas e científicas para o projeto e avaliação de interfaces de aplicativos móveis colaborativos.

Isso porque, além de caracterizar a proposta motivacional para uso contínuo do Waze, as estratégias identificadas neste trabalho podem ser utilizadas na melhoria e/ou no desenvolvimento de soluções que visam potencializar o uso contínuo de outros aplicativos móveis colaborativos, tanto para o contexto de aplicativos de navegação, quanto para outros domínios. Em termos científicos/metodológicos, os resultados do MIS reforçaram a aplicabilidade do método, devido à sua fundamentação teórica, para identificar as estratégias de design comunicadas na interface, que visam potencializar determinadas qualidades de uso, neste caso, as estratégias motivacionais para uso contínuo de softwares/aplicativos.

\section{Trabalhos Relacionados}

$\mathrm{Na}$ literatura, foram identificados alguns trabalhos que buscaram avaliar sistemas e/ou aplicativos colaborativos com o intuito de identificar estratégias de qualidades de uso (e.g., usabilidade, acessibilidade, comunicabilidade, colaboração, sociabilidade e gamificação) que guiaram seu design para potencializar o sucesso e a motivação na adoção do sistema.

Seguindo essa linha, Coutinho et al. (2011) aplicaram o MIS para identificar as estratégias sonoras utilizadas em jogos para orientar seus jogadores durante a interação, através de um estudo de caso no jogo FPS Half Life 2. Posteriormente, os autores 
verificaram o impacto dessas estratégias na acessibilidade de jogadores surdos. A pesquisa revelou que a adoção de recursos de áudio, como elemento estratégico para orientar jogadores durante a interação, pode comprometer a acessibilidade e o desempenho de jogadores surdos, quando não há opções alternativas para esse público, uma vez que esse tipo de usuário não poderá fazer uso dessa informação sonora durante a utilização do jogo.

O trabalho realizado por Barbosa et al. (2013) buscou analisar e caracterizar como o Facebook apoia a sociabilidade de seus membros. Para isso, o MIS foi utilizado com o objetivo de identificar as estratégias adotadas pelo projetista dessa rede social para prover sociabilidade aos seus usuários. Os resultados mostraram que o Facebook adota estratégias consideradas relevantes para promover uma interação social de qualidade em redes sociais online.

Já o trabalho realizado por Silva et al. (2014) investigou, através do MIS, a eficácia das estratégias de marketing na interface de sites de hotel, através de um estudo de caso no website do apart-hotel Promenade Champagnat. Finalmente, a pesquisa conduzida por Silva et al. (2015) buscou caracterizar como as estratégias de gamificação estavam sendo adotadas como fator motivacional em aplicativos educacionais. Os autores apresentaram um estudo de caso no aplicativo Duolingo, cujo foco é o ensino de idiomas, e através da aplicação do MIS demonstrou os benefícios de adotar a gamificação em aplicativos educacionais.

Os trabalhos apresentados nesta seção investigaram os benefícios de adotar qualidades de uso como fator motivacional para melhorar a experiência do usuário em sistemas/aplicativos colaborativos. Além disso, todos os autores argumentaram e enfatizaram a importância desse tipo de investigação para a melhoria e/ou desenvolvimento das diferentes categorias de sistemas/aplicativos. Contudo, a maioria dos trabalhos focou na caracterização da adoção de uma qualidade de uso específica e nenhum deles analisou a utilização dessas estratégias no contexto de aplicativos colaborativos de navegação.

Nesse sentido, o presente trabalho se difere dos demais, uma vez que apresenta uma caracterização de todas as estratégias de qualidade de uso que visam potencializar a utilização contínua de aplicativos colaborativos de navegação. Essa investigação é relevante, uma vez que esse tipo de aplicativo tem se tornado cada vez mais popular. Além disso, a identificação das estratégias adotadas, sob a perspectiva de diferentes qualidades de uso, pode contribuir para que projetistas de interface e pesquisadores compreendam melhor a aplicabilidade de cada qualidade de uso adotada como estratégia motivacional nesse contexto.

\section{Metodologia e Referencial Teórico}

Considerando o objetivo deste trabalho, a seguinte questão de pesquisa foi investigada: "Quais estratégias de qualidade de uso são utilizadas pelo Waze que contribuem para motivar o uso desse aplicativo?”. A metodologia adotada para responder essa questão consistiu em uma abordagem qualitativa, dividida em 3 etapas.

Inicialmente, foi realizado um levantamento bibliográfico com o objetivo de identificar diretrizes de qualidades de uso, presentes na literatura, que poderiam ser 
utilizadas como fator motivacional para uso contínuo de sistemas/aplicativos colaborativos. Em seguida, a interface do Waze foi inspecionada, seguindo os passos do Método de Inspeção Semiótica (MIS) [de Souza et al. 2006] para identificar e apresentar quais decisões foram tomadas pelo projetista do Waze para motivar seus usuários, e se essas decisões são compatíveis com as estratégias levantadas na literatura.

A terceira etapa buscou investigar junto aos usuários do Waze se as estratégias adotadas pelo aplicativo contribuem como fator motivacional para uso contínuo dessa aplicação. Para isso foi aplicado um questionário online e as respostas foram analisadas de forma a caracterizar a influência das qualidades de uso na utilização do Waze. A seguir, será descrito o conceito de qualidades de uso adotado neste trabalho. Posteriormente o MIS será apresentado, bem como a justificativa pela escolha desse método.

\subsection{Qualidade de Uso como fator motivacional para uso de sistemas/aplicativos}

A Experiência do Usuário (do inglês User Experience (UX)) está relacionada a como um indivíduo se sente enquanto usuário de um produto, sistema ou serviço, e ao prazer e à satisfação evocados no contato com esse artefato [Norman e Nielsen, 2013]. No âmbito de soluções tecnológicas a UX é potencializada por propriedades que caracterizam a qualidade de uso dos sistemas interativos [Norman e Nielsen, 2013].

Segundo Prates et al. (2007), a incorporação dessas propriedades pode variar de acordo com o objetivo e/ou domínio do sistema. Contudo, de forma geral, independente do sistema, para que o apoio computacional de um software e/ou aplicação seja satisfatório para o usuário, é desejável que o projetista comunique as suas intenções e concepções através da interface projetada (i.e., comunicabilidade), remova os obstáculos que impedem o usuário de acessar e interagir com o mesmo (i.e., acessibilidade) e torne o uso adequado aos usuários alvo da aplicação (i.e., usabilidade). Em outras palavras, o sistema deve prover, simultaneamente, as respectivas propriedades de qualidade de uso: comunicabilidade, acessibilidade e usabilidade [Prates et al. 2007].

Além dessas, que são consideradas essenciais para todos os tipos de sistemas/aplicativos, outras propriedades têm sido incorporadas como estratégias para motivar o uso contínuo dos diferentes domínios de sistemas/aplicativos. Por exemplo, a colaboração e a sociabilidade que têm sido potencializadas em sistemas colaborativos, e a gamificação, que tem sido utilizada em diferentes domínios de softwares para manter os usuários engajados [Pereira et al. 2010][de Oliveira et al. 2012][Zichermann e Cunningham, 2011].

Segundo Preece (2001) e Prates et al. (2007) a colaboração e a sociabilidade, referem-se às regras sociais, como privacidade, liberdade de expressão, confiança e outros aspectos que surgem da interação entre pessoas. Assim, sistemas colaborativos (e.g., redes sociais online, editores de conteúdo, blogs) com uma boa colaboração e sociabilidade, são aqueles que têm políticas colaborativas e sociais estabelecidas, que são compreensíveis por seus membros e, além disso, aceitáveis e suficientemente práticas para que possam ser implementadas. A gamificação, por sua vez, é caracterizada pela utilização dos princípios de design de jogos, como interação e colaboração, em um contexto que não trata especificamente de um jogo (e.g., profissional, social, educacional) para envolver usuários e auxiliar na resolução de desafios [Zichermann e Cunningham, 2011]. 
Todas essas propriedades visam maximizar a melhoria da UX, e por isso podem atuar como fatores motivacionais para que os usuários adotem e utilizem continuamente um aplicativo colaborativo. Uma forma de caracterizar se essas propriedades foram implementadas no projeto de interface e interação é através da avaliação da interface, que pode ser conduzida através do Método de Inspeção Semiótica, o MIS, que será apresentado na próxima seção.

\subsection{Método de Inspeção Semiótica}

O Método de Inspeção Semiótica (MIS) é baseado na teoria da Engenharia Semiótica (EngSem), uma teoria explicativa de Interação Humano Computador (IHC), que permite entender os fenômenos envolvidos no design, uso e avaliação de um sistema interativo [de Souza et al. 2006].

A avaliação utilizando o MIS consiste em cinco etapas [de Souza et al. 2006]: (1) inspeção dos signos metalinguísticos; (2) inspeção dos signos estáticos; (3) inspeção dos signos dinâmicos; (4) contraste e comparação entre as mensagens identificadas em cada uma das inspeções e (5) apreciação da qualidade da metacomunicação. Nos passos de 1 a 3, o especialista deve ser capaz de reconstruir a metamensagem proposta pelo projetista a partir dos signos inspecionados. No passo 4, o especialista contrasta as mensagens geradas nos passos anteriores e, a partir desta análise, explora a possibilidade do usuário atribuir significados diferentes a um mesmo objeto (signo). Finalmente, no passo 5, o especialista gera uma versão unificada da metamensagem e registra a sua apreciação final da qualidade do sistema.

Embora originalmente o MIS tenha sido proposto para avaliar a comunicabilidade de um sistema, uma revisão na literatura realizada por Reis e Prates (2011), revelou que, devido à sua fundamentação teórica, esse método também pode ser utilizado para identificar as estratégias de design comunicadas na interface que visam potencializar determinadas qualidades de uso.

Neste sentido, trabalhos como o realizado por Coutinho et al. (2011) identificaram estratégias sonoras para orientação em jogos, através da aplicação do MIS. Já o trabalho realizado por Barbosa et al. (2011) utilizou o MIS para identificar estratégias de sociabilidade em redes sociais. Silva et al. (2014), utilizaram o MIS para identificar estratégias de marketing em sites de hotel, e Silva et al. (2015) aplicou o MIS para identificar estratégias de gamificação em aplicativos educacionais.

Demonstrada a aplicabilidade do MIS nesses contextos, a adoção desse método para avaliar o aplicativo Waze permitirá, por meio da apreciação da metamensagem do designer, identificar estratégias de qualidade de uso comunicadas na interface desse aplicativo para manter os usuários motivados em seu uso contínuo. A seguir, apresentamos os principais resultados da primeira etapa dessa pesquisa.

\section{Apreciação da Proposta de Projeto do Waze para Motivar seu Uso Contínuo}

Nesta seção, é apresentada a análise da interface do Waze, realizada utilizando o MIS, indicando a proposta do projetista para potencializar (i.e., motivar) o uso contínuo desse aplicativo, bem como as estratégias de qualidade de uso adotadas por ele. A avaliação foi realizada no período de três semanas, durante o mês de setembro de 2015 , e foi conduzida 
por dois autores desse trabalho, dos quais um já possui mais de 05 anos de experiência na aplicação do MIS no contexto de avaliação de sistemas colaborativos. O escopo da avaliação contemplou os principais cenários de uso do Waze, como: (1) traçar a rota para um destino; (2) inserir alertas; (3) troca de mensagens entre os contatos e (4) editar o perfil. A seguir, os principais resultados dessa etapa são apresentados.

\subsection{Proposta do Waze para motivar seu uso contínuo}

A partir da análise do MIS verificou-se que a intenção do projetista em relação ao Waze é disponibilizar um aplicativo cujo objetivo é fornecer a melhor rota para um determinado destino, através da contribuição de uma comunidade composta por usuários em trânsito (e.g., motoristas e passageiros). Além disso, o Waze visa proporcionar uma experiência mais prazerosa e dinâmica, através da colaboração entre os usuários que compartilham informações de trânsito e tráfego durante sua utilização (e.g., alertas de congestionamentos, acidentes, pontos de interdição, radares, pontos de fiscalização, preços em postos de gasolina, atualização de dados no mapa, entre outras).

Para atingir seu objetivo principal, o Waze estimula a colaboração entre os usuários e, consequentemente, sua utilização contínua fazendo uso de estratégias como: (1) a criação de uma rede de contatos para interação; (2) bate papo entre os contatos; (3) o estabelecimento de um ranking da interação, (4) a oferta de níveis de evolução de permissão e (4) a oferta de recompensas por colaboração. Em relação ao ranking, o usuário avança nas posições na medida em que acumula pontos por utilizar as funcionalidades do aplicativo. O Waze permite que seus colaboradores compartilhem suas posições no ranking de modo que possam estabelecer competições.

Em relação aos níveis de evolução de permissão, os mesmos são divididos em Waze bebê, adulto, guerreiro, cavaleiro e rei, que também são alcançados em função do acúmulo de pontos por utilização do aplicativo. Na medida em que evolui, o usuário recebe determinadas vantagens, como por exemplo, a possibilidade de editar um mapa. Os pontos são obtidos a partir da criação de alertas, da distância percorrida utilizando o Waze e/ou da atualização de mapas (e.g., inserindo nome nas ruas, número às edificações). De forma complementar, o Waze oferece recompensas, que são conquistadas a partir do momento que o usuário atende um conjunto de critérios, como, por exemplo, adicionar o primeiro amigo.

Outra forma de incentivar a interação entre os usuários, proposta pelo projetista do Waze, consiste na possibilidade de integrar o uso do aplicativo com as redes sociais, como Facebook e Twitter, de modo que seja possível: (1) localizar contatos dessas redes para adicioná-los no Waze, (2) compartilhar o percurso, o local atual e/ou o horário estimado de chegada ao destino. Além disso, o Waze oferece aos usuários a opção de enviar mensagens aos contatos. Essas mensagens podem ser públicas (i.e., para todos os contatos), privadas (i.e., para um contato ou um grupo específico) e/ou um "Beep Beep", que consiste em um tipo de mensagem onde um usuário chama atenção de outro. A Figura 1 ilustra evidências da proposta do projetista para a interface do Waze. 

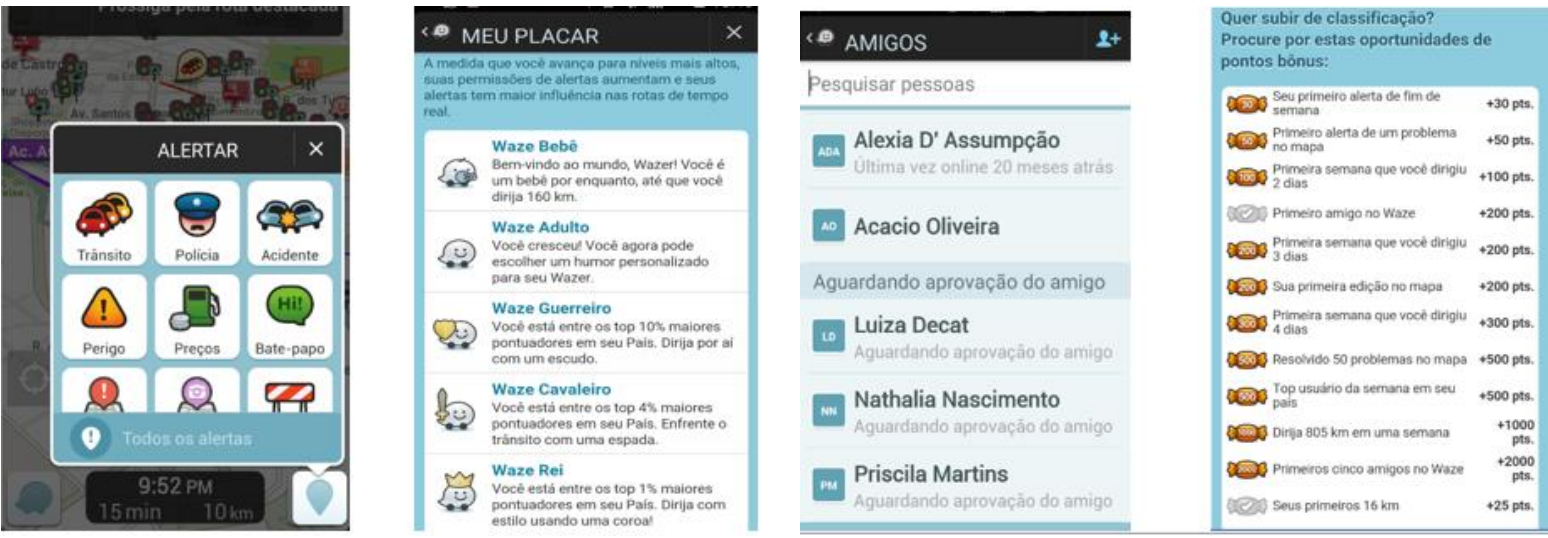

Figura 1 - Proposta do projetista do Waze

\subsection{Estratégias para Motivar o Uso Contínuo do Waze}

Uma vez identificada a proposta do projetista do Waze para motivar seu uso contínuo, este trabalho buscou verificar se as decisões comunicadas na interface desse aplicativo são compatíveis com as estratégias que, de acordo com a literatura [Nielsen, 1994][de Souza e Preece, 2004][Pontes, 2008][Furtado et al. 2010][Werbach e Hunter, 2012] [Knoll, 2014][W3C, 2016], podem ser consideradas diretrizes de qualidades de uso que contribuem para manter os usuários motivados durante a interação em ambientes colaborativos. A Tabela 1 lista 43 estratégias levantadas na literatura, subdivididas em 6 qualidades de uso.

Tabela 1 - Estratégias do projeto de interface e interação para motivar uso contínuo de sistemas colaborativos identificadas na literatura

\begin{tabular}{|c|c|c|c|}
\hline Qualidade de Uso & Cód. & Estratégia (Diretrizes)* & Autor(es) \\
\hline \multirow{9}{*}{ Sociabilidade } & S1 & Propósitos bem definidos & [de Souza e Preece, 2004] \\
\hline & $\mathrm{S} 2$ & Considerar as diferenças de cada usuário & [de Souza e Preece, 2004] \\
\hline & S3 & Políticas bem definidas & [de Souza e Preece, 2004] \\
\hline & S4 & $\begin{array}{l}\text { Permitir que as pessoas desenvolvam suas } \\
\text { próprias identidades online }\end{array}$ & [de Souza e Preece, 2004] \\
\hline & S5 & $\begin{array}{l}\text { Encorajar a empatia e confiança entre os } \\
\text { membros }\end{array}$ & [de Souza e Preece, 2004] \\
\hline & S6 & Encorajar a reciprocidade & [de Souza e Preece, 2004] \\
\hline & S7 & Manter discussões em evidência & [de Souza e Preece, 2004] \\
\hline & S8 & Diversificar possibilidades de comunicação & [de Souza e Preece, 2004] \\
\hline & S9 & Garantir proteção e privacidade & [de Souza e Preece, 2004] \\
\hline \multirow{6}{*}{ Colaboração } & $\mathrm{C} 1$ & Participação do usuário como colaborador & [Furtado et al. 2010] \\
\hline & $\mathrm{C} 2$ & Compreensão dos objetivos & [Furtado et al. 2010] \\
\hline & $\mathrm{C} 3$ & Incentivar a participação coletiva & [Furtado et al. 2010] \\
\hline & $\mathrm{C} 4$ & Acompanhamento do progresso individual & [Furtado et al. 2010] \\
\hline & C5 & $\begin{array}{l}\text { Quantificação da contribuição do usuário no } \\
\text { objetivo }\end{array}$ & [Furtado et al. 2010] \\
\hline & C6 & Configuração de características colaborativas & [Furtado et al. 2010] \\
\hline \multirow{5}{*}{ Gamificação } & G1 & Múltiplos caminhos & [Werbach e Hunter, 2012] \\
\hline & G2 & Orientar o usuário na interação & [Werbach e Hunter, 2012] \\
\hline & G3 & Níveis de evolução & [Werbach e Hunter, 2012] \\
\hline & G4 & Erro como aprendizado & [Werbach e Hunter, 2012] \\
\hline & G5 & Competição e colaboração & [Werbach e Hunter, 2012] \\
\hline
\end{tabular}




\begin{tabular}{|c|c|c|c|}
\hline & G6 & Diversão & [Werbach e Hunter, 2012] \\
\hline & G7 & Incorporar a narrativa ao jogo & [Werbach e Hunter, 2012] \\
\hline & G8 & Definir metas & [Werbach e Hunter, 2012] \\
\hline & G9 & Estabelecer rankings & [Werbach e Hunter, 2012] \\
\hline & G10 & Reconhecimento de padrões & [Werbach e Hunter, 2012] \\
\hline & G11 & Permitir coleções & [Werbach e Hunter, 2012] \\
\hline & G12 & Surpresa e prazer inesperado & [Werbach e Hunter, 2012] \\
\hline & G13 & Presentes / Bonificação & [Werbach e Hunter, 2012] \\
\hline & G14 & Reconhecimento de uma realização & [Werbach e Hunter, 2012] \\
\hline & G15 & Informar status de progresso & [Werbach e Hunter, 2012] \\
\hline \multirow{7}{*}{ Usabilidade } & U1 & Visibilidade do sistema & $\begin{array}{l}\text { [Nielsen, 1994][Knoll, } \\
\text { 2014] }\end{array}$ \\
\hline & $\mathrm{U} 2$ & Minimização da carga de memória & $\begin{array}{l}\text { [Nielsen, 1994][Knoll, } \\
\text { 2014] }\end{array}$ \\
\hline & $\mathrm{U} 3$ & Facilidade de acesso & $\begin{array}{l}\text { [Nielsen, 1994][Knoll, } \\
\text { 2014] }\end{array}$ \\
\hline & U4 & Ajuda e documentação & $\begin{array}{l}\text { [Nielsen, } \\
\text { 2014] }\end{array}$ \\
\hline & U5 & Bom aproveitamento do espaço da tela. & $\begin{array}{ll}\text { [Nielsen, } & 1994][\text { Knoll, } \\
2014] & \\
\end{array}$ \\
\hline & U6 & Interação incentivadora & $\begin{array}{l}\text { [Nielsen, } \\
\text { 2014] }\end{array}$ \\
\hline & U7 & Configuração persistente & $\begin{array}{l}\text { [Nielsen, 1994][Knoll, } \\
2014]\end{array}$ \\
\hline Comunicabilidade & $\mathrm{C} 1$ & Explicitar caminhos de interação & [Pontes, 2008] \\
\hline \multirow{2}{*}{$\begin{array}{l}\text { Comunicabilidade, } \\
\text { Acessibilidade e } \\
\text { Usabilidade }\end{array}$} & M1 & $\begin{array}{l}\text { Contextualização ao usuário e Adequação às } \\
\text { Funcionalidades }\end{array}$ & $\begin{array}{l}\text { [Pontes, 2008][W3C, 2016] } \\
\text { [Nielsen, } \\
\text { 2014] }\end{array}$ \\
\hline & M2 & Tratamento de erros e auxílio ao usuário. & $\begin{array}{l}\text { [Pontes, 2008][W3C, 2016] } \\
\text { [Nielsen, } \\
\text { 2014] }\end{array}$ \\
\hline $\begin{array}{c}\text { Comunicabilidade } \\
\text { e Usabilidade }\end{array}$ & M3 & Feedback claro, imediato e informativo. & $\begin{array}{l}\text { [Pontes, 2008][Nielsen, } \\
\text { 1994][Knoll, 2014] }\end{array}$ \\
\hline \multirow{2}{*}{$\begin{array}{l}\text { Acessibilidade e } \\
\text { Usabilidade }\end{array}$} & M4 & Acessibilidade diversa e facilitada & $\begin{array}{l}{[\text { W3C, 2016] [Nielsen, }} \\
\text { 1994][Knoll, 2014] }\end{array}$ \\
\hline & M5 & Previsibilidade e consistência & $\begin{array}{l}{\left[\begin{array}{ll}\text { W3C, 2016] } \\
\text { 1994] [Nielsen, }\end{array}\right] \text { Knoll, 2014] }}\end{array}$ \\
\hline
\end{tabular}

Posteriormente, conforme demonstrado na Tabela 2, foi realizado um mapeamento entre as decisões do projetista do Waze e as estratégias motivacionais identificadas a partir da literatura. É importante ressaltar que uma estratégia pode refletir em uma ou mais decisões do projetista da interface. Além disso, na Tabela 2 foi utilizada a coluna "Cod. Estratégias" para referenciar as diretrizes de qualidade de uso apresentadas na Tabela 1.

Tabela 2 - Estratégias motivacionais versus Decisões do projetista do Waze

\begin{tabular}{|c|c|c|c|c|}
\hline $\begin{array}{c}\text { Cod. } \\
\text { Estratégias }\end{array}$ & \multicolumn{2}{|c|}{$\begin{array}{c}\text { Cod. e Decisões do } \\
\text { Projetista }\end{array}$} & Exemplo de aplicação no Waze & Qualidade de Uso \\
\hline $\mathrm{C} 1$ & \multirow{6}{*}{ D1 } & \multirow{6}{*}{$\begin{array}{l}\text { Oferecer diferentes } \\
\text { tipos de alerta }\end{array}$} & \multirow{6}{*}{$\begin{array}{l}\text { O Waze disponibiliza a possibilidade de gerar } \\
\text { alertas sobre: congestionamentos, acidentes, } \\
\text { pontos de interdição, radares, preços em postos } \\
\text { de gasolina, atualizações no mapa, entre outros. } \\
\text { Estes alertas permitem aos usuários colaborar } \\
\text { entre si no alcance do objetivo final, que é a } \\
\text { determinação da melhor rota. }\end{array}$} & Colaboração \\
\hline $\mathrm{C} 3$ & & & & Colaboração \\
\hline G5 & & & & Gamificação \\
\hline S5 & & & & Sociabilidade \\
\hline S6 & & & & Sociabilidade \\
\hline S8 & & & & Sociabilidade \\
\hline
\end{tabular}




\begin{tabular}{|c|c|c|c|c|}
\hline $\mathrm{C} 3$ & \multirow{3}{*}{ D2 } & \multirow{3}{*}{$\begin{array}{l}\text { Oferecer pontos por } \\
\text { atividades } \\
\text { executadas. }\end{array}$} & \multirow{3}{*}{$\begin{array}{l}\text { Durante a interação com as funcionalidades do } \\
\text { Waze (e.g., solicitar rota, informar alerta, editar } \\
\text { mapa, adicionar um contato) o usuário recebe } \\
\text { pontos que permite posicioná-lo em um ranking } \\
\text { e o habilita para receber recompensas. }\end{array}$} & Colaboração \\
\hline G5 & & & & Gamificação \\
\hline G14 & & & & Gamificação \\
\hline G12 & \multirow{4}{*}{ D3 } & \multirow{4}{*}{$\begin{array}{l}\text { Oferecer } \\
\text { recompensas por } \\
\text { uma conquista. }\end{array}$} & \multirow{4}{*}{$\begin{array}{l}\text { O Waze oferece recompensas a partir do } \\
\text { momento que o usuário atende um conjunto de } \\
\text { critérios e alcança uma conquista, como, por } \\
\text { exemplo, adicionar o primeiro amigo. }\end{array}$} & Gamificação \\
\hline G13 & & & & Gamificação \\
\hline G14 & & & & Gamificação \\
\hline G6 & & & & Gamificação \\
\hline G3 & \multirow[b]{4}{*}{ D4 } & \multirow{4}{*}{$\begin{array}{ll}\text { Oferecer } & \text { níveis } \\
\text { evolução } & \text { de } \\
\text { permissão } & \end{array}$} & \multirow{4}{*}{$\begin{array}{l}\text { Durante a interação com as funcionalidades do } \\
\text { Waze, o usuário acumula pontos e pode evoluir } \\
\text { seu nível de permissão no aplicativo (e.g., Waze } \\
\text { bebê, adulto, guerreiro, cavaleiro e rei). Quanto } \\
\text { mais evoluído, maior as possibilidades de } \\
\text { interação com o aplicativo e intervenção na } \\
\text { definição de rotas por parte do usuário. }\end{array}$} & Gamificação \\
\hline G5 & & & & Gamificação \\
\hline $\mathrm{C} 4$ & & & & Colaboração \\
\hline U6 & & & & Usabilidade \\
\hline G1 & \multirow{3}{*}{ D5 } & \multirow{3}{*}{$\begin{array}{l}\text { Oferecer além da } \\
\text { digitação, a opção } \\
\text { de comando de voz. }\end{array}$} & \multirow{3}{*}{$\begin{array}{l}\text { O Waze disponibiliza a opção de realizar a } \\
\text { busca por pontos de interesse, através digitação } \\
\text { e comando de voz. }\end{array}$} & Gamificação \\
\hline $\mathrm{C} 1$ & & & & Comunicabilidade \\
\hline M4 & & & & $\begin{array}{l}\text { Acessibilidade e } \\
\text { usabilidade }\end{array}$ \\
\hline $\mathrm{C} 3$ & \multirow{6}{*}{ D6 } & \multirow{6}{*}{$\begin{array}{l}\text { Oferece a opção de } \\
\text { pesquisar/adicionar/ } \\
\text { interagir com } \\
\text { amigos. }\end{array}$} & \multirow{6}{*}{$\begin{array}{l}\text { Durante a interação com as funcionalidades do } \\
\text { Waze, o usuário pode interagir com times (i.e., } \\
\text { grupos de contatos), adicionar e pesquisar por } \\
\text { contatos, e se comunicar com eles através de } \\
\text { mensagens públicas ou privadas. }\end{array}$} & Colaboração \\
\hline G6 & & & & Gamificação \\
\hline S5 & & & & Sociabilidade \\
\hline S6 & & & & Sociabilidade \\
\hline S7 & & & & Sociabilidade \\
\hline S8 & & & & Sociabilidade \\
\hline $\mathrm{U} 2$ & \multirow{3}{*}{ D7 } & \multirow{3}{*}{$\begin{array}{l}\text { Armazenar } \\
\text { preferências } \\
\text { usuário }\end{array}$} & \multirow{3}{*}{$\begin{array}{l}\text { O Waze armazena automaticamente uma lista } \\
\text { de destinos recentemente percorridos pelo } \\
\text { usuário. Estes e outros destinos podem ser } \\
\text { salvos como favoritos. Isso facilita a busca e } \\
\text { recuperação de destinos favoritos. }\end{array}$} & Usabilidade \\
\hline U3 & & & & Usabilidade \\
\hline U7 & & & & Usabilidade \\
\hline U4 & \multirow[b]{2}{*}{ D8 } & \multirow[b]{2}{*}{$\begin{array}{l}\text { Oferecer opção de } \\
\text { ajuda }\end{array}$} & \multirow{2}{*}{$\begin{array}{l}\text { O aplicativo disponibiliza ao usuário a opção de } \\
\text { Ajuda para especificar problemas mais comuns } \\
\text { e como resolvê-los. Além disso, o Waze oferece } \\
\text { caminhos múltiplos para acesso a Ajuda. }\end{array}$} & Usabilidade \\
\hline G1 & & & & Gamificação \\
\hline G5 & \multirow{4}{*}{ D9 } & \multirow{4}{*}{$\begin{array}{lr}\text { Estabelecer } & \text { um } \\
\text { ranking } & \text { de } \\
\text { interação } & \end{array}$} & \multirow{4}{*}{$\begin{array}{l}\text { O Waze exibe um ranking de pontuação, com a } \\
\text { classificação dos usuários. O ranking pode ser } \\
\text { compartilhado de modo que os usuários } \\
\text { estabeleçam competições entre si. }\end{array}$} & Gamificação \\
\hline G9 & & & & Gamificação \\
\hline G15 & & & & Gamificação \\
\hline $\mathrm{C} 4$ & & & & Colaboração \\
\hline S2 & \multirow{2}{*}{ D10 } & \multirow{2}{*}{$\begin{array}{l}\text { Oferecer } \\
\text { configurações de } \\
\text { acordo com o perfil } \\
\text { do usuário }\end{array}$} & \multirow{2}{*}{$\begin{array}{l}\text { O usuário pode configurar o idioma padrão, } \\
\text { unidades de medida (milhas ou quilômetros), } \\
\text { alterar o design de seu carro de acordo com sua } \\
\text { preferencia e alterar o esquema de cores do } \\
\text { mapa. }\end{array}$} & Sociabilidade \\
\hline S4 & & & & Sociabilidade \\
\hline C6 & \multirow{2}{*}{ D11 } & \multirow{2}{*}{$\begin{array}{l}\text { Oferecer } \\
\text { configurações de } \\
\text { interação. }\end{array}$} & O usuário pode ativar ou desativar bate-papos & Colaboração \\
\hline S9 & & & e conexões com redes sociais. & Sociabilidade \\
\hline M2 & D12 & $\begin{array}{l}\text { Recalcular rotas } \\
\text { quando o usuário }\end{array}$ & $\begin{array}{l}\text { O aplicativo calcula, automaticamente, uma } \\
\text { nova rota para o usuário, quando o mesmo se }\end{array}$ & $\begin{array}{c}\text { Comunicabilidade, } \\
\text { acessibilidade e } \\
\text { usabilidade. }\end{array}$ \\
\hline G4 & & & & Gamificação \\
\hline
\end{tabular}


A Figura 2 evidencia alguns exemplos de decisões do projetista do Waze que são referenciadas através da coluna "Cód. e Decisões do Projetista", da Tabela 2.
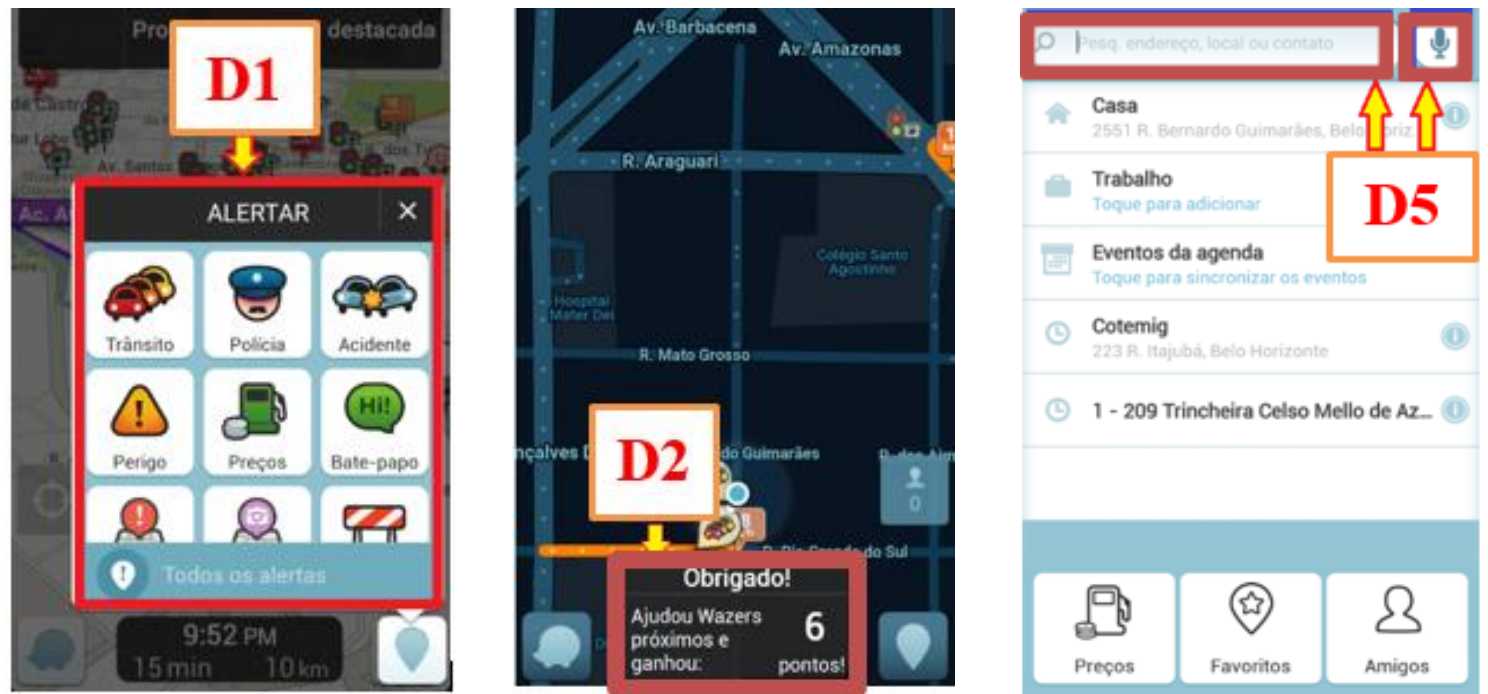

Figura 2 - Interface do Waze com evidências das decisões do projetista

Ao comparar a proposta do Waze, identificada através da avaliação com o MIS, com as estratégias motivacionais identificadas na literatura, foi possível constatar que as decisões do projetista do Waze estão alinhadas com as diretrizes de qualidades de uso que segundo Nielsen (1994), de Souza e Preece (2004), Pontes (2008), Furtado et al. (2010), Werbach, e Hunter (2012), Knoll (2014), W3C (2016), podem favorecer a utilização contínua do aplicativo e potencializar a experiência satisfatória dos usuários.

Para um melhor entendimento desses resultados, verificou-se que o projetista adotou $67 \%$ das estratégias identificadas na literatura. Além disso, não foi observado o uso de novas estratégias, ou seja, todas as decisões do projetista, observadas através do MIS, são compatíveis com pelo menos uma diretriz de qualidade de uso encontrada na literatura. Esse trabalho também buscou verificar a incidência de uma determinada qualidade de uso no projeto de interface e interação do Waze. Dessa forma, o gráfico da Figura 3 indica a frequência em que uma mesma qualidade de uso foi representada, através da implementação de suas diretrizes, no Waze.

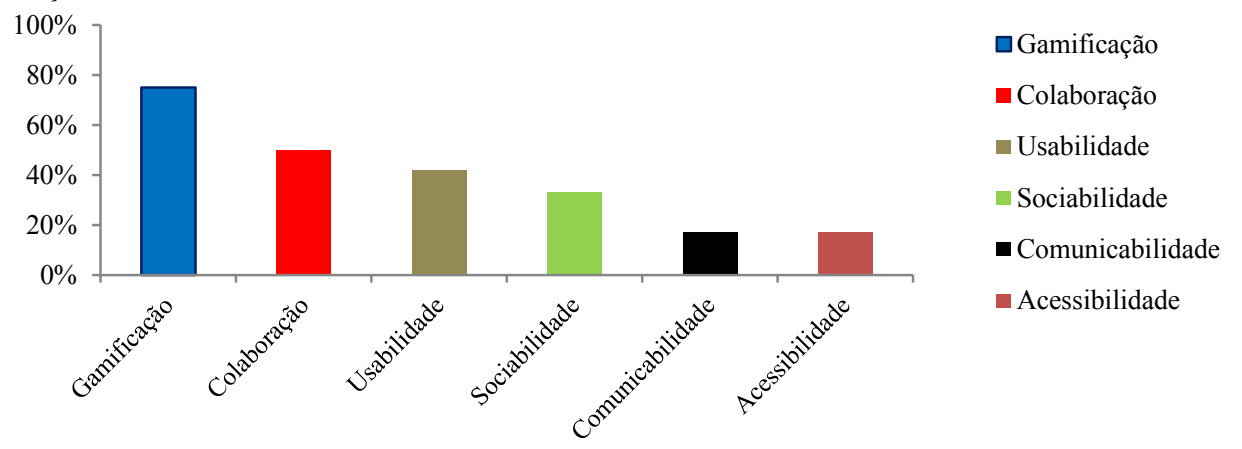

Figura 3 - Incidência das qualidades de uso nas decisões do projetista do Waze

A partir dos dados obtidos foi possível observar que o projetista do Waze adotou 
estratégias que contemplam todas as qualidades de uso que visam potencializar a experiência satisfatória do usuário. Porém, houve uma predominância das seguintes propriedades: gamificação, colaboração, usabilidade e sociabilidade que, além da qualidade da interação, buscam motivar o uso prazeroso e contínuo do aplicativo.

\section{Análise da Motivação de uso do Waze sob a perspectiva dos Usuários}

Com o objetivo de investigar junto aos usuários do Waze se as estratégias de colaboração, sociabilidade, gamificação, usabilidade, acessibilidade e comunicabilidade adotadas pelo projetista do Waze contribuem como fator motivacional para uso desse aplicativo, foi aplicado um questionário online com 13 questões de múltipla escolha para os usuários do Waze. As questões foram definidas e agrupadas de modo a identificar: (1) o perfil dos participantes da pesquisa, (2) se eles se sentem motivados ao utilizar o Waze e (3) quais os fatores que mais contribuem para essa motivação. A aplicação do questionário ocorreu no período de 2 semanas, durante a segunda quinzena do mês de setembro de 2015. O mesmo foi divulgado através de redes sociais, e-mails e fóruns de discussões sobre o aplicativo.

Ao todo 185 pessoas participaram da pesquisa, respondendo ao questionário. Em relação ao perfil dos participantes $10 \%$ foram classificados na faixa etária entre 13 e 20 anos, a maioria (51\%) declarou estar na faixa entre 21 e 28 anos, $20 \%$ na faixa de 29 a 36 anos, 14\% na faixa de 37 a 56 anos e 5\% acima de 56 anos. É importante destacar que a participação de usuários menores de 18 anos é válida, uma vez que o aplicativo pode ser utilizado não apenas por motoristas, mas também por passageiros (i.e., qualquer usuário em trânsito). Quanto ao tipo de aplicativos que utilizam, onde mais de uma categoria poderia ser marcada, $100 \%$ dos participantes afirmam utilizar aplicativos de navegação, $78 \%$ declararam utilizar redes sociais, 31\% utilizam aplicativos de jogos, 97\% fazem uso de aplicativos de mensagens instantâneas, 66\% utilizam aplicativos de serviços (e.g., banco, taxi) e 5\% fazem uso de aplicativos de outras categorias.

\subsection{Análise das Motivação dos Usuários}

Inicialmente, os participantes foram convidados a responder sobre as motivações iniciais que os levavam a escolher o Waze como aplicativo de navegação (i.e., o que motivou a fazer download do aplicativo). Nessa questão, mais de uma opção poderia ser marcada. $\mathrm{O}$ resultado obtido mostra que a maioria dos participantes $(57 \%)$ escolheu usar o Waze pelo fato do aplicativo ser gratuito e 37\% começaram a utilizá-lo por recomendações de amigos.

Além disso, os participantes também foram questionados sobre a frequência semanal de uso do Waze, e após fazerem o download do aplicativo, se os mesmos sentiamse motivados durante sua utilização. Os resultados demonstraram, que mais da metade dos entrevistados, $59 \%$, utilizam o Waze pelo menos cinco vezes por semana, e que $72 \%$ dos participantes se sentem motivados a fazer uso contínuo do aplicativo. A Figura 4 apresenta os fatores que mais motivam os usuários dessa pesquisa a utilizaram o Waze continuamente. Esses fatores correspondem às decisões do projeto de interface e interação do Waze listadas na Tabela 2. Nessa questão, mais de um fator poderia ser indicado, porém, não era obrigatória.

Através da Figura 4 é possível constatar que, embora os participantes pudessem optar por não responder essa questão, ou ainda marcar a opção "Nenhum dos Fatores", as 
decisões do projetista do Waze, que refletem em estratégias de qualidades de uso para potencializar a experiência do usuário e manter o uso contínuo da aplicação, contribuem como fatores motivacionais para a utilização do aplicativo.

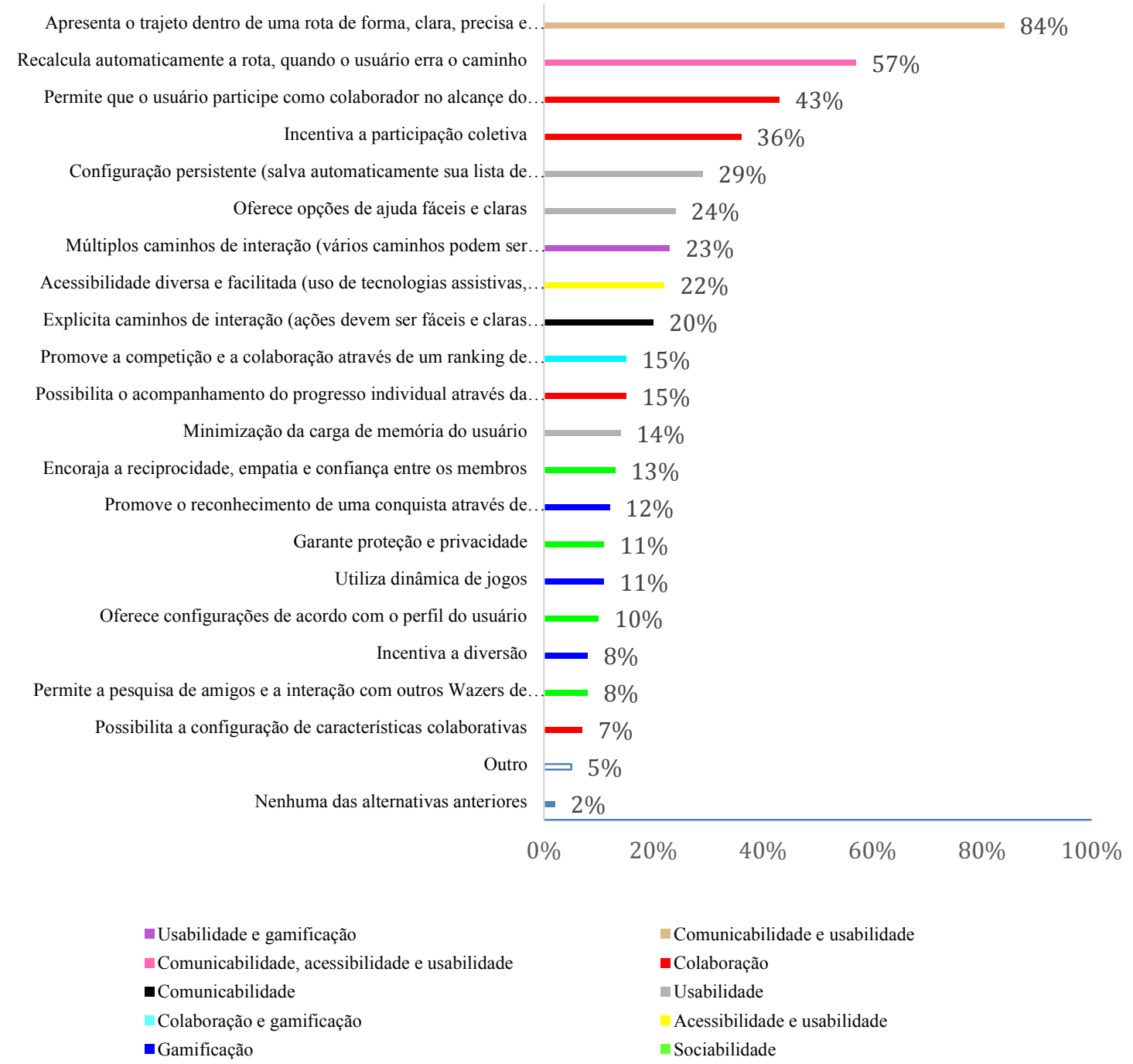

Figura 4 - Fatores motivacionais para uso do Waze. Os fatores (i.e., as estratégias motivacionais) estão agrupados por qualidade de uso conforme a legenda da figura.

Conforme observado no gráfico da Figura 4, as estratégias de usabilidade e colaboração, identificadas na interface do Waze, se destacam em relação aos fatores que mais motivam os usuários na utilização contínua do aplicativo. Entretanto, é importante ressaltar que, em alguns casos, essas qualidades de uso apareceram associadas a outras, como, por exemplo, a estratégia mais referenciada pelos participantes: "Apresenta o trajeto de uma rota de forma, clara, precisa e objetiva", engloba características de comunicabilidade e usabilidade.

Em seguida, os entrevistados foram questionados, explicitamente, se características como colaboração, sociabilidade e gamificação contribuem como fatores motivacionais 
para a utilização contínua do Waze. Os resultados indicaram que para $87 \%$ dos participantes a colaboração contribui para que eles se mantenham engajados no uso do aplicativo. Isso reforça o argumento de que a colaboração é uma qualidade de uso que favorece o engajamento dos usuários no uso de aplicativos de navegação. Além disso, 67\% afirmaram que as características de sociabilidade também influenciam positivamente no uso continuo do Waze. Já a utilização de dinâmica de jogos (i.e., a gamificação) foi apontada por $42 \%$ dos participantes como fator motivacional. De forma complementar, no questionário foram feitas perguntas que buscaram verificar a satisfação do usuário em relação ao aplicativo. Os resultados indicaram que $94 \%$ dos participantes pretendem continuar utilizando o Waze como aplicativo de navegação.

\section{Discussão dos Resultados}

O Waze é um dos principais aplicativos colaborativos de navegação, com mais de 50 milhões de usuários e classificado, de acordo com Google Play (2016), com uma média de 4,6 estrelas (em uma escala máxima de 5 estrelas). Diante dessa aceitação, esse trabalho buscou caracterizar as estratégias de qualidade de uso adotadas pelo projetista desse aplicativo que contribuem como fator motivacional para uso contínuo do Waze.

Por meio da análise do MIS, foi possível verificar que o projetista utiliza elementos conhecidos no âmbito das qualidades de uso. Tais elementos podem ser vistos como estratégias que o Waze adota para motivar os usuários e que podem contribuir para mantêlos engajados na utilização continua do aplicativo. Os resultados obtidos, por meio da pesquisa com os usuários, sustentam o argumento de que as estratégias identificadas na interface do Waze exercem influência motivacional para uso contínuo do aplicativo.

Nesse sentido, esta pesquisa evidencia a importância de adotar estratégias de qualidade de uso como, comunicabilidade, acessibilidade, usabilidade, colaboração, sociabilidade e gamificação, das quais, sobressaíram a usabilidade e a colaboração, no projeto de interface e modelo de interação de aplicativos colaborativos de navegação. Tal adoção contribui, assim, para que essas propriedades se consolidem como importantes estratégias para potencializar o engajamento e a experiência satisfatória dos usuários no uso desse tipo de aplicação.

\section{Conclusões e Trabalhos Futuros}

Este trabalho buscou apreciar e caracterizar as estratégias de qualidades de uso adotadas em aplicativos colaborativos de navegação e a contribuição das mesmas para o uso contínuo desse tipo de aplicação. Para isso foi realizado um estudo de caso no Waze e os resultados apresentados permitiram constatar que o projetista desse aplicativo adota estratégias consideradas relevantes para promover um ambiente colaborativo que mantém o usuário motivado a interagir continuamente.

Em termos de contribuições, apesar de apresentar um estudo de caso no Waze, a apreciação realizada se faz relevante tanto em termos técnicos, quanto científicos/metodológicos para a área. Isso porque, em termos científicos/metodológicos, os resultados do MIS reforçaram a aplicabilidade do método, devido à sua fundamentação teórica, para identificar as estratégias de design comunicadas na interface, que visam 
potencializar determinadas qualidades de uso, neste caso, as estratégias motivacionais para uso contínuo de softwares/aplicativos. Já em termos técnicos, as estratégias apresentadas neste trabalho podem ser utilizadas na melhoria e/ou no desenvolvimento de soluções que visam potencializar o uso contínuo de outros aplicativos móveis colaborativos, tanto para o contexto de aplicativos de navegação, quanto para outros domínios.

Como trabalhos futuros, um ponto interessante a ser investigado seria a possibilidade de identificar componentes na interface (i.e., signos), que permitem classificar as estratégias motivacionais oferecidas pelos aplicativos. Isso ajudaria no projeto e avaliação de outros aplicativos/sistemas que buscam manter seus usuários motivados em sua utilização contínua.

\section{Referências}

Barbosa, G. A. R.; Santos, G, E.; and Victor M. de O. Pereira, V. M. O. 2013. Caracterização qualitativa da sociabilidade no Facebook. In Proc. IHC '13, p. 72-81.

Britto, T.C., Martins, L.C.G., Penteado, B.E. e Anacleto, J.C. 2011. Técnicas de Prototipação para Smartphones no Apoio à Avaliação de Interfaces com o Usuário. In Proc. IHC\&CLIHC'11, p. 39- 42.

Brown, Q., Bonsignore, E., Hatley, L., Druin, A., Walsh, G., Foss, E., Brewer, R., Hammer, J. e Golub, E. 2010. Clear Panels: A Technique to Design Mobile Application Interactivity. In Proc. DIS'10, p. 360-363.

Coutinho, F.; Prates, R. O.; Chaimowicz, L. 2011. An analysis of information conveyed through audio in an fps game and its impact on deaf players experience. In Proc. of SBGAMES'11. p. 53-62.

de Oliveira, D. H. D., de Miranda, L. C., de Miranda, E. E. C., da Silva,L. F. 2012. Prototipação de interfaces de aplicativos para dispositivos móveis: estado da arte e desafios de IHC. In Proc. of IHC '12, p. 315-324.

de Souza, C. S., Leitão, C. F., Prates, R. O., and da Silva, E. J. 2006. The semiotic inspection method. In: Proc. of IHC'06, p. 148-157.

de Souza, C. S., Leitão, C. F., Prates, R. O., Bim, S.A., da Silva, E.J. 2010. Can inspection methods generate valid new knowledge in HCI? The case of semiotic inspection. In: International Journal of Human-Computer Studies, 68(1-2), p. 22-40.

de Souza, C.S. and Preece, J. 2004. A Framework for Analyzing and Understanding Online Communities. In: Interacting with Computers, v. 16(3), p. 579-610.

Forlizzi, J. and Battarbee, K. 2004. Understanding experience in interactive systems. In Proc. of DIS '04, p. 261-268.

Furtado, V. et al. 2010. Apoio ao projeto de sites colaborativos: uma abordagem fundamentada na associação de requisitos de colaboração e de interação com prépadrões de interface. In Proc. of IHC'10.

Google Play. 2016. Disponível em: <https://play.google.com/store/apps>. 
Knoll, R. C. Desenvolvimento de heurísticas de usabilidade para tablets. Caderno de Estudos Tecnológicos, FATEC, Bauru, Julho 2014. V.2, n.1, p. 93-109.

Nielsen, J. Usability engineering. Elsevier, 1994.

Norman, D. and Nielsen, J. 2013. The Definition of User Experience. Disponível em: $<$ https://goo.gl/Jx1bZj>. Último acesso em: 29 de março de 2016.

O Globo. 2013. Waze já tem 6 milhões de usuários no Brasil, que é o $2^{\circ}$ mercado do app. Disponível em: <http://goo.gl/xImxvq>. Último acesso em: 25 de Março de 2016.

Pereira, R.; Baranauskas, M. C. C. \& da Silva, S. R. P. 2010. Softwares sociais: uma visão orientada a valores. In Proc. of IHC '10, p. 149-158.

Pontes, P. E. 2008. Especificação de requisitos para comunicabilidade em websites na engenharia semiótica. 108 p. Faculdade de Informática do Centro Universitário Ritter dos Reis, Porto Alegre, 2008.

Prates, R. O. and Barbosa, S. D. J. 2007. Introdução à Teoria e Prática da Interação Humano Computador fundamentada na Engenharia Semiótica. In: T. Kowaltowski e K. K. Breitman (Org.). Sociedade Brasileira de Computação. SBC. Rio de Janeiro.

Preece, J. 2001. Online communities: Usability, Sociability, Theory and Methods. In R. Earnshaw, R. Guedj, A. van Dam and T. Vince (Eds) Frontiers of Human-Centred Computing, Online Communities and Virtual Environments.

Reis, S. de S. and Prates, R. O. 2011. Applicability of the Semiotic Inspection Method: a Systematic Literature Review. In: Proc. of X IHC \& V CLIHC'11, p. 177-186.

Silva, M. R., Coutinho, F. R. S. and Oliveira, A. P. G. 2014. Estratégias de comunicação de marketing para o website corporativo do Promenade Champagnat: usando o MIS para o turismo. In Proc. of IHC'14, p. 333-336.

Silva, J. C. R.; Oliveira, E, R; and Barbora, G. A. R. 2015. Caracterização de Estratégias de Gamificação em Aplicativos Móveis Educacionais: Um Estudo de Caso do Aplicativo Duolingo. In Proc. of IHC '15.

Tarouco, F. 2013. A metrópole comunicacional e a popularização dos apps para dispositivos móveis. Seminário Internacional de Pesquisa em Comunicação. Epistemologia e desafios da pesquisa no campo da comunicação, 2013.

W3C. 2016. Designing for inclusion. 2012. Disponível em: <http:/goo.gl/dJX65Y>. Último acesso em: 25 de Março de 2016

Werbach, K.; Hunter, D. For The Win: How Game Thinking Can Revolutionize Your Business. Filadélfia, Pensilvânia: Wharton Digital Press. 148 p., 2012.

Waze. 2016. Disponível em: <https://www.waze.com/>. Último acesso em: 25 de Março de 2016

Zichermann, G.; Cunningham, C. 2011. Gamification by Design - Implementing Game Mechanics in Web and Mobile Apps. (1st ed.). O'Reilly Media, Inc. 